\title{
Sick Newborn
}

National Cancer Institute

\section{Source}

National Cancer Institute. Sick Newborn. NCI Thesaurus. Code C81291.

A neonate that is unwell. 